\title{
PREDICTION OF CLIMATE CHANGE USING ARIMA MODEL
}

\author{
D Hebsiba beula ${ }^{1}$, S Srinivasan ${ }^{2}$ C D Nanda Kumar ${ }^{3}$ \\ ${ }^{1}$ Research Scholar, Department Mathematics \& Actuarial Science, B S Abdur Rahman \\ Crescent Institute of Science \& Technology, Chennai, 600048, India, \\ ${ }^{2}$ Professor, Department Mathematics \& Actuarial Science, B S Abdur Rahman Crescent \\ Institute of Science \& Technology, Chennai, 600048, India \\ ${ }^{3}$ Associate Professor, Department Mathematics \& Actuarial Science, B S Abdur Rahman \\ Crescent Institute of Science \& Technology, Chennai, 600048, India, \\ Email: 'hebsiba_maths_2018@crescent.education, ${ }^{2}$ srinivasan@crescent.education, \\ ${ }^{3}$ prof.cdnandakumar@gmail.com
}

\begin{abstract}
:
The climate and weather system prediction has always attracted interest. Climate change risks including physical risks, liability risks and transition risks, it's directly affecting the insurance industry. Climate change is majorly affecting the insurance sector; they are such as extreme heat during summer and extreme rainfall (Flood). It affects both insurance and reinsurance sector. Constructing the model is a necessary process but choosing the model which suits our data is very necessary. In those days the weather reports telecast in news but now even our smart phone notified the weather. In this paper study the climate prediction algorithms using $R$ and also using Cost-free $R$ language tool to forecast the climate using time ARIMA model for the Indian climate.
\end{abstract}

Key words: Climate change, insurance, prediction, ARIMA Model

\section{Introduction:}

Climate Change is real. What makes it worse is because of the human activities that are not environment friendly, in the example using fossil fuel for the vehicle, coal-powered generator, cement production, etc. One of the effects of these activities is increasing in temperature and depletion on the ozone layer. Before we build the model, we have to know the data first. How it looks, how different does the pattern they are. I will describe what the data set really is and what the information contains on it. The 2019-20 Australian bushfires killed more than a billion animals and caused more than $\$ 4.4$ billion in damage. Climate-linked issues, such as extreme heat, natural disasters, and biodiversity loss and the failure to respond to these challenges in time dominate reports issued by organizations such as the World Economic Forum.

At first glance, the effects of climate change may not seem detrimental to property and casualty $(\mathrm{P} \& \mathrm{C})$ insurers. They can use the annual policy cycle and their sophisticated understanding of evolving risks to reprice and rearrange portfolios to avoid long-term exposure to climate events. 
And the growth in the value at risk and possibly volatility should increase the demand for new and different insurance solutions and services, which, in turn, could expand the industry's opportunities. Insurers, however, must be careful not to underestimate the true threat of climate change. Because its effects are systemic, climate risk is likely to stress local economies and more grimly cause market failures that affect both consumers and insurers. More frequent catastrophic events, in combination with the need to meet evolving regulatory requirements, can threaten company business models and make insuring some risk unaffordable for customers or unfeasible for insurers.

\section{Methodology:}

In this paper the climate prediction did with Autoregressive Integrated Moving Average (ARIMA) model for time series data, is a forecasting algorithm based on the idea that the information in the past values of the time series can alone be used to predict the future values.

\section{Data Analysis}

Actuaries, statisticians and many other professionals are increasingly engaged in analyzing and interpreting large data sets, in order to determine whether there is any relationship between variables, and to assess the strength of that relationship. Exploratory data analysis (EDA) is the process of analyzing data to gain further insight into the nature of the data, its patterns and relationships between the variables, before any formal statistical techniques are applied. That is we approach the data free of any pre-conceived assumptions or hypotheses. We first see the patterns in the data before we impose any views on it and fit models.

Here we have 56 years of past data from 1961 to 2016 for temperature, rainfall, crop production and the area of land used by agriculture in INDIA. The dataset are shown below:

\begin{tabular}{|c|c|c|c|c|}
\hline Year & Temperatı & infall & Area of lar & p prodı \\
\hline 1961 & 24 & 116.61 & 17.4952 & 34.95 \\
\hline 1962 & 24.04 & 99.82 & 17.6482 & 34.64 \\
\hline 1963 & 24.15 & 101.75 & 17.6154 & 35.66 \\
\hline 1964 & 24.1 & 103.69 & 17.6712 & 36.61 \\
\hline 1965 & 24.07 & 78.94 & 17.7177 & 34.47 \\
\hline 1966 & 24.36 & 88.18 & 17.753 & 34.15 \\
\hline 1967 & 24.11 & 96.16 & 17.7807 & 36.78 \\
\hline 1968 & 23.94 & 88.28 & 17.8364 & 38.59 \\
\hline 1969 & 24.46 & 95.67 & 17.7792 & 39.94 \\
\hline 1970 & 24.26 & 104.59 & 17.7817 & 42.44 \\
\hline 1971 & 23.91 & 101.42 & 17.7946 & 43.02 \\
\hline 1972 & 24.1 & 78.92 & 17.8751 & 40.6 \\
\hline 1973 & 24.41 & 101.63 & 17.8751 & 44.16 \\
\hline 1974 & 24 & 87.93 & 17.9282 & 42.24 \\
\hline 1975 & 23.74 & 107.9 & 17.9694 & 47.05 \\
\hline 1976 & 24.07 & 94.28 & 17.9649 & 46 \\
\hline 1977 & 24.35 & 105.82 & 17.9591 & 50.24 \\
\hline 1978 & 24.24 & 103.11 & 18.0592 & 52.4 \\
\hline 1979 & 24.57 & 85.83 & 18.058 & 48.82 \\
\hline 1980 & 24.55 & 98.53 & 18.0272 & 50.43 \\
\hline 1981 & 24.27 & 97.54 & 18.0401 & 54.02 \\
\hline 1982 & 24.15 & 90.38 & 18.0771 & 52.74 \\
\hline 1983 & 24.12 & 110.07 & 18.0462 & 59.44 \\
\hline 1984 & 24.26 & 96.73 & 18.1452 & 60.31 \\
\hline 1985 & 24.45 & 95.41 & 18.134 & 61.21 \\
\hline 1986 & 24.2 & 94.8 & 18.1192 & 61.16 \\
\hline 1987 & 24.57 & 90.73 & 18.1543 & 59.85 \\
\hline 1988 & 24.42 & 111.84 & 18.1091 & 67 \\
\hline 1989 & 24.03 & 93.94 & 18.1501 & 71.58 \\
\hline
\end{tabular}

\begin{tabular}{|c|c|c|c|c|}
\hline Year & Temper & Rainfall & Area of la & rop prodi \\
\hline 1989 & 24.03 & 93.94 & 18.1501 & 71.58 \\
\hline 1990 & 24.21 & 116.78 & 18.1413 & 72.59 \\
\hline 1991 & 24.28 & 97.52 & 18.1586 & 73.68 \\
\hline 1992 & 24.15 & 91.88 & 18.1305 & 77.07 \\
\hline 1993 & 24.43 & 100.65 & 18.1382 & 79.74 \\
\hline 1994 & 24.46 & 107.94 & 18.1291 & 82.07 \\
\hline 1995 & 25.29 & 103.54 & 18.0945 & 83.68 \\
\hline 1996 & 24.55 & 98.58 & 18.0589 & 87.07 \\
\hline 1997 & 24.1 & 98.59 & 18.0981 & 88.84 \\
\hline 1998 & 24.76 & 100.73 & 18.0874 & 90.18 \\
\hline 1999 & 24.67 & 93.05 & 18.1021 & 94.32 \\
\hline 2000 & 24.6 & 86.28 & 18.0975 & 92.08 \\
\hline 2001 & 24.73 & 92.11 & 18.0487 & 94.78 \\
\hline 2002 & 25 & 81.83 & 18.056 & 84.74 \\
\hline 2003 & 24.72 & 103.63 & 18.0249 & 96.97 \\
\hline 2004 & 24.74 & 90.04 & 18.0375 & 94.12 \\
\hline 2005 & 24.58 & 100.68 & 18.0126 & 99.9 \\
\hline 2006 & 25.06 & 96.81 & 17.9906 & 105.98 \\
\hline 2007 & 24.77 & 98.28 & 17.962 & 116.23 \\
\hline 2008 & 24.61 & 93.18 & 17.9757 & 117.28 \\
\hline 2009 & 25.11 & 79.48 & 18.0068 & 112.83 \\
\hline 2010 & 25.13 & 101.3 & 17.9573 & 124.91 \\
\hline 2011 & 24.67 & 93.03 & 17.967 & 134.07 \\
\hline 2012 & 24.69 & 87.89 & 17.9642 & 136.19 \\
\hline 2013 & 24.82 & 103.55 & 17.9698 & 141.74 \\
\hline 2014 & 24.73 & 83.61 & 17.9721 & 144.36 \\
\hline 2015 & 24.91 & 87.52 & 17.9721 & 139.6 \\
\hline 2016 & 26.45 & 88.03 & 17.9721 & 142.8 \\
\hline
\end{tabular}




\section{Relationship between the temperature, rainfall, crop production and area of land used by agriculture}

First import the ggplot2 package in $\mathrm{r}$ and then import the required data for analysis. Then we can use the ggplot graph for our data. Insert our temperature, rainfall, crop production and area of land (in sq mtr lakhs) and we can use the different color for dataset. What we can conclude is the crop production (Yellow color) is continuous increasing and the rainfall data (Blue color) is stationary see in figure 2. The temperature (Red color) is increased in some period and decreased in some period and the area of land (Green color) is lower than 1980s'when compared to 1960s' but now the land used for agriculture is slowly decreased shown in figure 3.

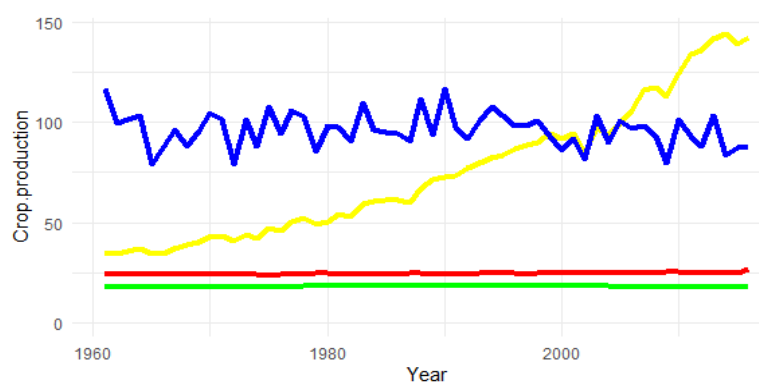

Figure 2: Plots of temperature, rainfall, crop production, area of land

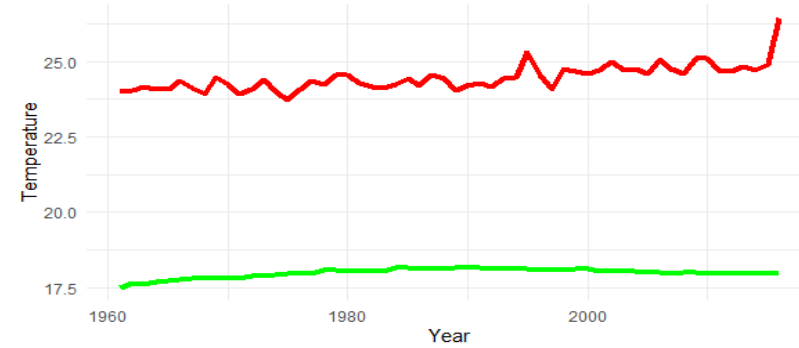

Figure 3: Plots of temperature and crop production

\section{The Kendall rank correlation coefficient}

Kendall's rank correlation coefficient $\tau$ measures the strength of dependence of rank correlation between two variables. Like the Spearman rank correlation coefficient, the Kendall rank correlation coefficient considers only the relative values of the bivariate data, and not their actual values. It is far more intensive from a calculation viewpoint, however, since it considers the relative values of all possible pairs of bivariate data, not simply the rank of $X_{i}$ and $Y_{i}$ for a given $i$. Let $n_{c}$ be the number of concordant pairs, and let $n_{d}$ be the number of discordant pairs. Assuming that there are no ties, the Kendall coefficient $\tau$ is defined as:

$$
\tau=\frac{\frac{n_{c}-n_{d}}{n(n-1)}}{2}
$$




\begin{tabular}{|c|c|c|}
\hline Variables & Correlation value & Results \\
\hline Year and Crop production & 0.9697759 & $\begin{array}{c}\text { The year and crop production move } \\
\text { together in the same direction but } \\
\text { there is not a direct relationship. }\end{array}$ \\
\hline Temperature and Crop production & 0.7278388 & $\begin{array}{l}\text { The temperature and crop production } \\
\text { move together in the same direction } \\
\text { but there is not a direct relationship. }\end{array}$ \\
\hline Rainfall and Crop production & 0.0668009 & $\begin{array}{l}\text { The rainfall and crop production move } \\
\text { together in the same direction but } \\
\text { there is not a direct relationship. }\end{array}$ \\
\hline Area of land and Crop production & 0.3961773 & $\begin{array}{l}\text { The area of land and crop production } \\
\text { move together in the same direction } \\
\text { but there is not a direct relationship. }\end{array}$ \\
\hline
\end{tabular}

\section{Table 1: Correlation table}

The dependent variable is crop production and the independent variables are year, temperature, rainfall and the area of land.

Linear correlation between a pair of variables looks at the strength of the linear relationship between them. The diagram below shows the various degree of positive correlation:

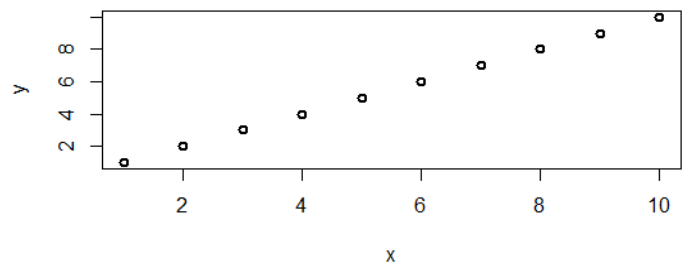

Figure 4: Perfect positive correlation

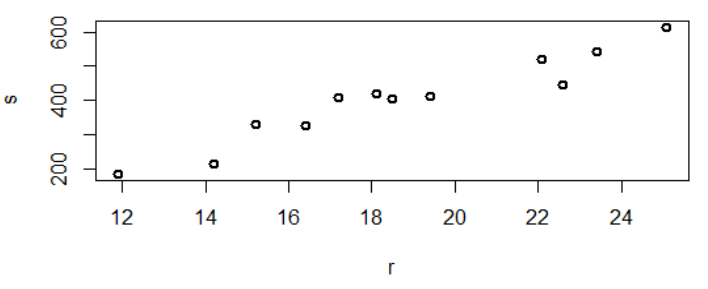

Figure 5: Strong positive correlation

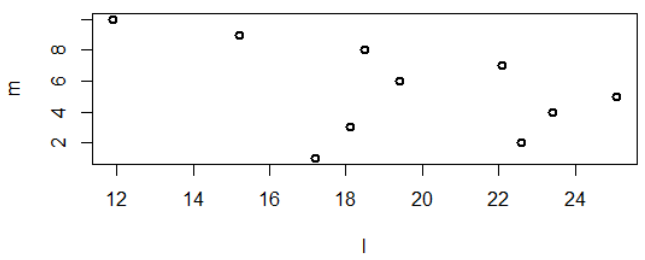

Figure 6: Weak positive correlation

For multivariate data sets with large dimensionality various techniques such as cluster analysis and principle components analysis (also called factor analysis) can be used to reduce the complexity of the data set. 


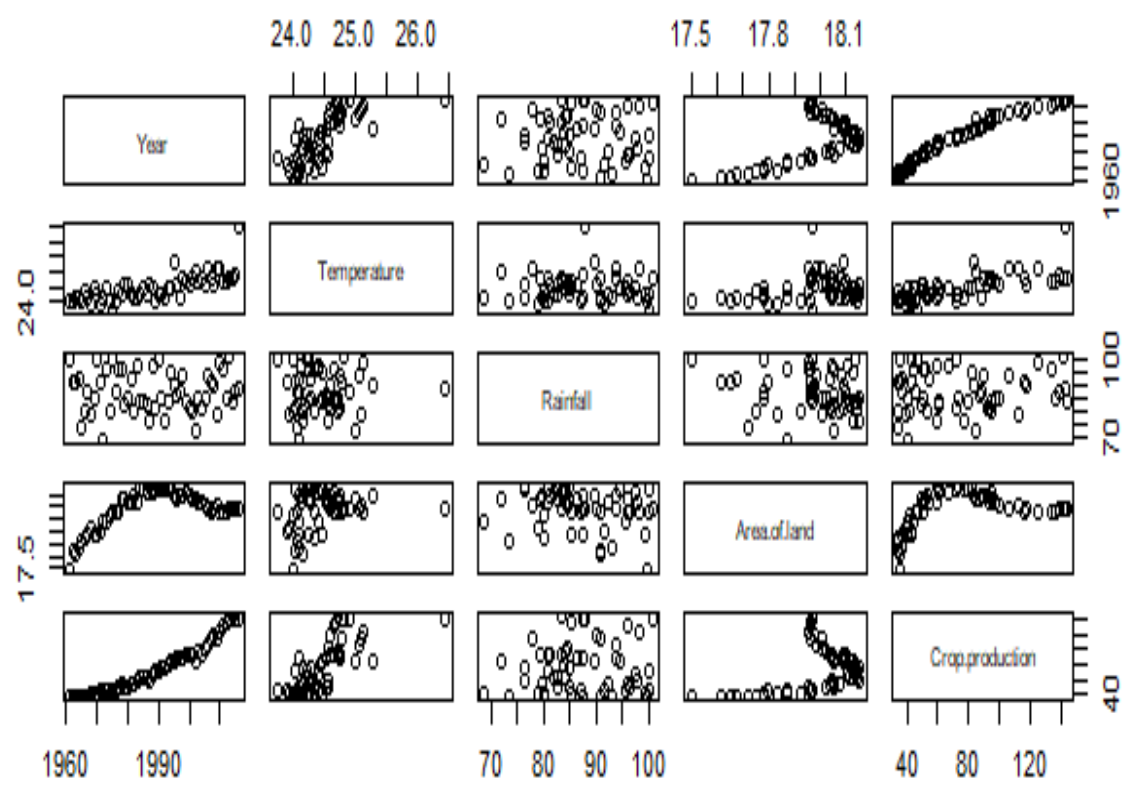

Figure 7: Relationship plots of Year, Temperature, Rainfall, and Area of land and Crop production

\section{Temperature Forecasting}

Import the temperature dataset in RStudio to predict the future temperature of India for next 25 years. And check the class of data, if the dataset are not in time series change the data into time series. Import some packages like forecast and t series; it's useful for forecasting in R.

\begin{tabular}{|c|c|}
\hline Minimum & 23.74 \\
\hline $1^{\text {st }}$ Quarter & 24.14 \\
\hline Median & 24.43 \\
\hline Mean & 24.47 \\
\hline $3^{\text {rd }}$ Quarter & 24.70 \\
\hline Maximum & 26.45 \\
\hline
\end{tabular}

Plot the data in auto plot function (figure 8), we can check the data is stationary or nonstationary. If the data is non stationary convert the data into stationary and then proceed to next step. Our temperature data is non stationary we can find by using the Augmented Dickey-Fuller test shortly called as adf.test. Here we the p-value is 0.1225 is greater than 0.05 so, the data is non-stationary. 


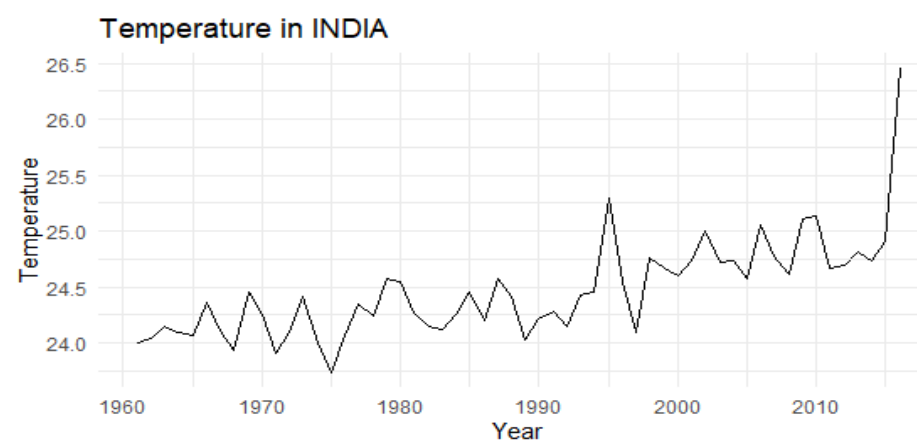

Figure 8: Graph of temperature

We can detect that the data is stationary or not using the ACF (Autocorrelation Function) and PACF (Partial Autocorrelation Function). If the graph is above and below the blue line, the data is non-stationary.

Series temp ts

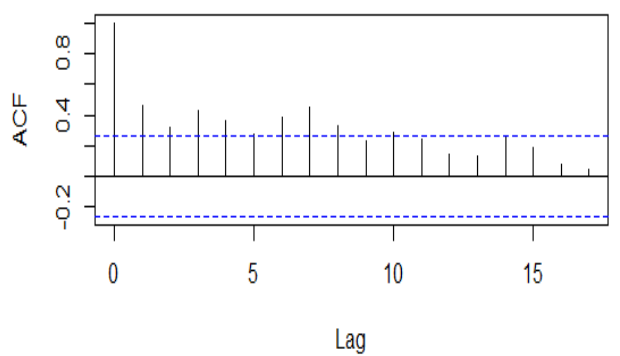

Figure 9: ACF of temperature
Series temp_ts

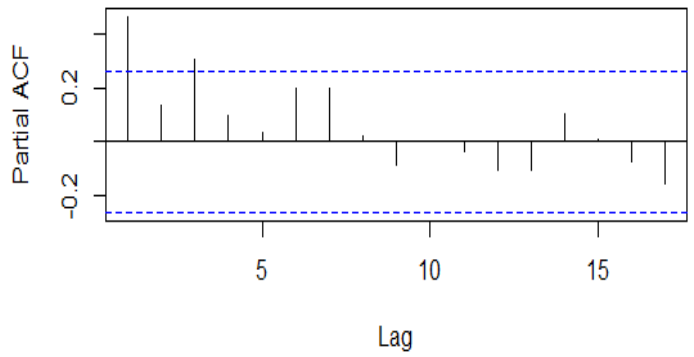

Figure 10: PACF of temperature

In above figure 9 and 10 we saw the ACF and PACF are above than blue lines. So differentiate the data and check the adf test and also the ACF and PACF. Now calculate the autocorrelation function and partial autocorrelation function of residuals, the lines are below the blue dotted line (figure $11 \& 12$ ).

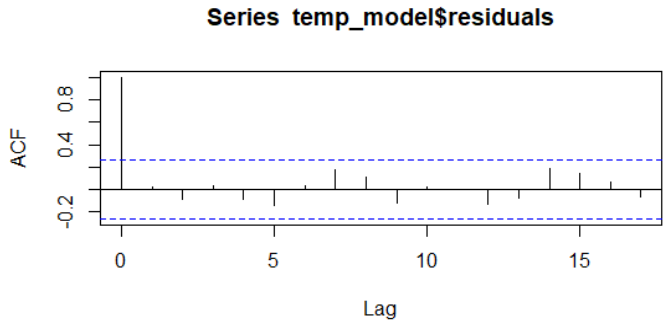

Figure 11: ACF of temp residuals

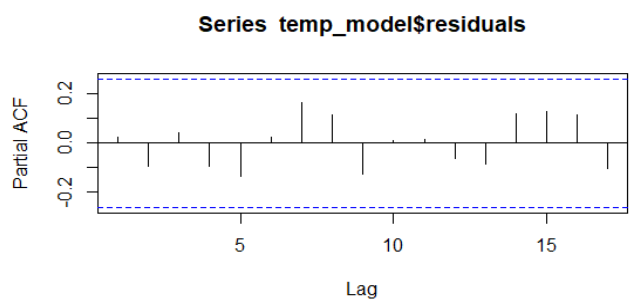

Figure 12: PACF of temp residuals

Again we calculate the adf test to see the data is stationary or not. Here we have a p-value is 0.04584 so, the data is stationary. Plot the graph to see the result (figure 13). If the data is stationary means the mean and variance are constant throughout the period. And use the forecast in build function to predicting the future. 


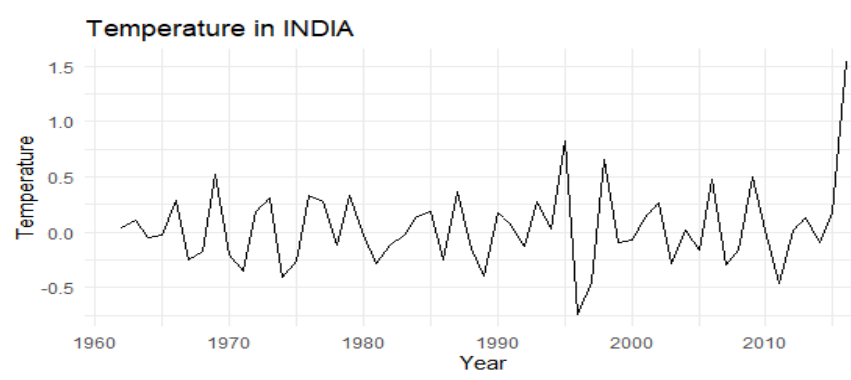

Figure 13: Plot of temperature (Stationary)

R Code:

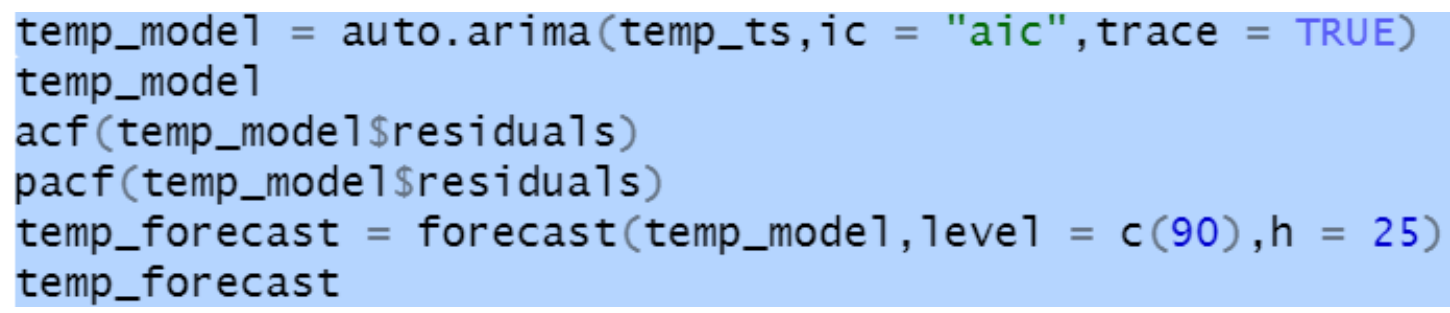

We can use the auto Arima function to calculate the $\mathrm{p}, \mathrm{d}$ and $\mathrm{q}$. Suppose we manually calculate the $p, d$ and $q$ means the aic and bic values are higher when compared to auto Arima function. The Akaike Information Criteria (AIC) and Bayesian Information Criteria (BIC) values are least value when compared to others. That means the $\mathrm{p}, \mathrm{d}$, q values are best fit to predicting the future. We use the 90 per cent level of significant to forecasting for next 25 years (figure 14). Here we take the $\mathrm{p}, \mathrm{d}$, q value is $(2,1,1)$ and the AIC value is 34.1 and BIC value is 44.13.

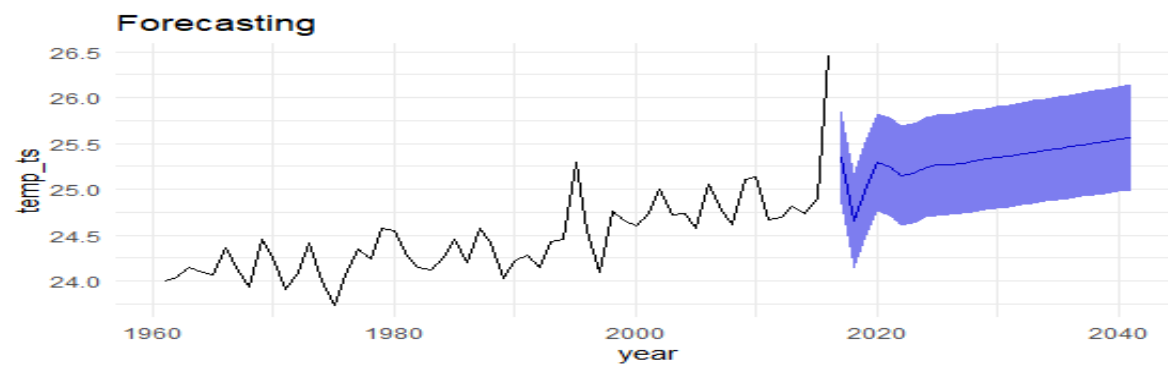

Figure 14: Temperature forecasting

We conclude that the year is increases the temperature of India is also increases.

\section{Rainfall Forecasting}

Import the rainfall dataset in RStudio to predict the future rainfall of India for next 25 years. And check the class of data, if the dataset are not in time series change the data into time series.

\begin{tabular}{|c|c|}
\hline Minimum & 78.92 \\
\hline $1^{\text {st }}$ Quarter & 90.30 \\
\hline Median & 96.77 \\
\hline
\end{tabular}




\begin{tabular}{|c|c|}
\hline Mean & 96.38 \\
\hline $3^{\text {rd }}$ Quarter & 101.66 \\
\hline Maximum & 116.78 \\
\hline
\end{tabular}

Plot the data in graph format (figure 15), we can check the data is stationary or non-stationary. If the data is non stationary convert the data into stationary and then proceed to next step. Our rainfall data is stationary we can find by using the Augmented Dickey-Fuller test shortly called as adf.test. The p-value is 0.02231 lower than 0.05 means the data is stationary. If the data is stationary means the mean and variance are constant.

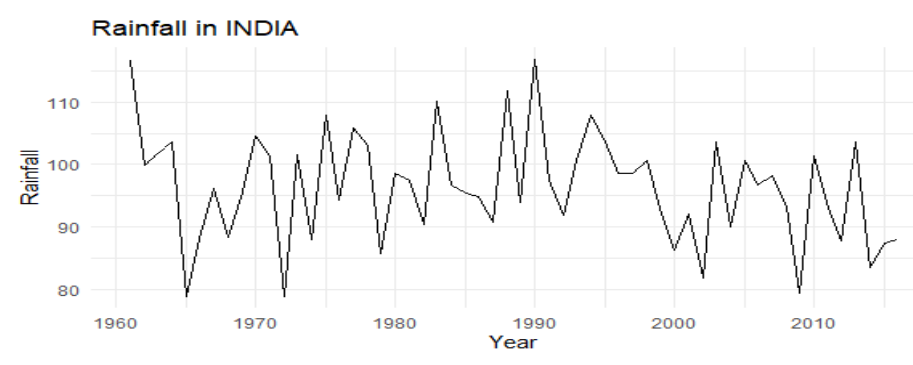

Figure 15: Graph of rainfall in India

R Code:

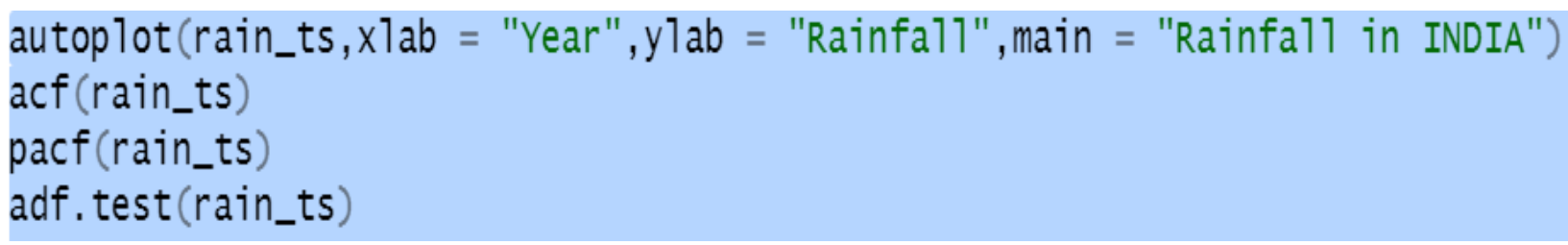

And then we calculate the autocorrelation function and partial autocorrelation function (figure 16 \& 17) to cross check the data is stationary or not and also calculate the $p, d, q$ value. But our rainfall data is stationary, so the $\mathrm{d}$ values are zero.

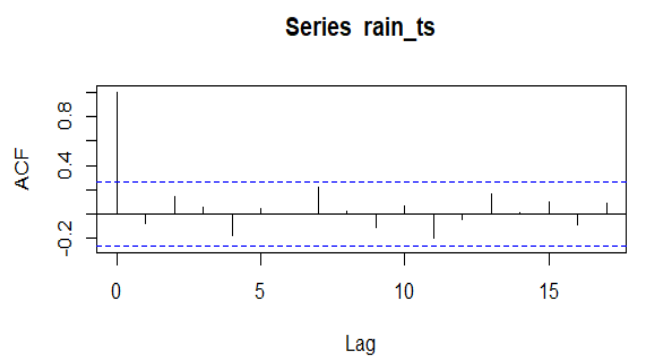

Figure 16: ACF of rainfall

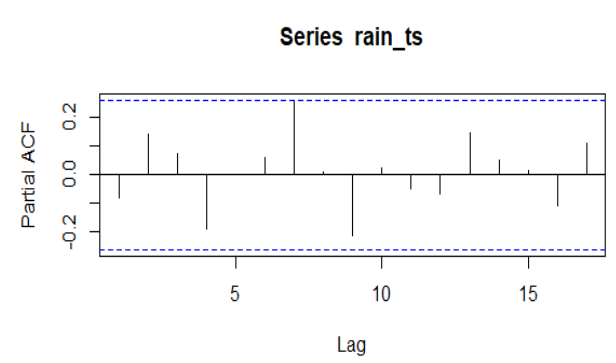

Figure 17: PACF of rainfall

We can use the auto Arima function to calculate the $\mathrm{p}, \mathrm{d}$ and $\mathrm{q}$. Suppose we manually calculate the $p, d$ and $q$ means the aic and bic values are higher when compared to auto Arima function. The Akaike Information Criteria (AIC) and Bayesian Information Criteria (BIC) values are least value when compared to others. That means the $p, d, q$ values are best fit to predicting the 
future. The AIC value is 407.69 is the best fit for forecasting when compared to others. We use the 90 per cent level of significant to forecasting for next 25 years (figure 18).

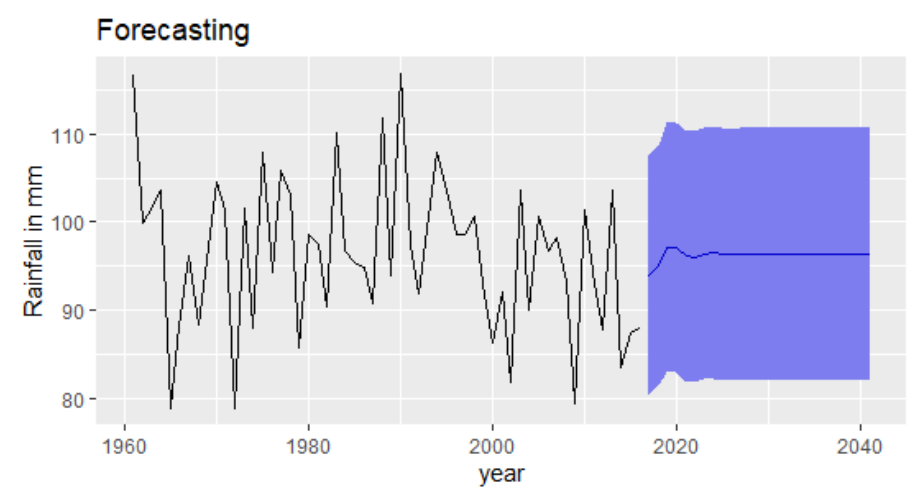

Figure 18: Rainfall Forecasting

R Code:

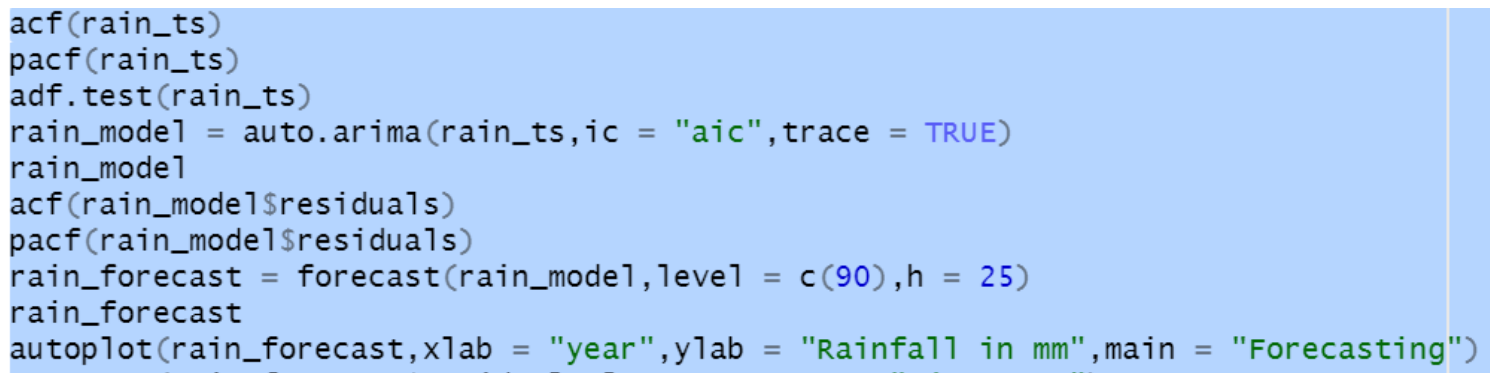

\section{Crop Production Forecasting}

Import the crop production dataset in RStudio to predict the future crop production of India for next 25 years. And check the class of data, if the dataset are not in time series change the data into time series. Import some packages like forecast and time series; it's useful for forecasting in $\mathrm{R}$.

\begin{tabular}{|c|c|}
\hline Minimum & 34.15 \\
\hline $1^{\text {st }}$ Quarter & 45.54 \\
\hline Median & 69.29 \\
\hline Mean & 74.79 \\
\hline $3^{\text {rd }}$ Quarter & 94.44 \\
\hline Maximum & 144.36 \\
\hline
\end{tabular}

Plot the data in auto plot function (figure 19), we can check the data is stationary or nonstationary. If the data is non stationary convert the data into stationary and then proceed to next step. Our crop production data is non stationary we can find by using the Augmented DickeyFuller test shortly called as adf.test. The p-value is 0.8595 greater than 0.05 means the data is non-stationary. 


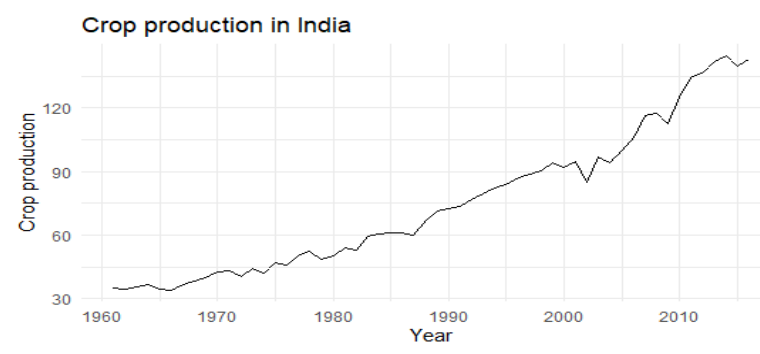

Figure 19: Plot of Crop production index

We can detect that the data is stationary or not using the ACF (Autocorrelation Function) and PACF (Partial Autocorrelation Function). If the graphs are above and below the blue line, the data is non-stationary.

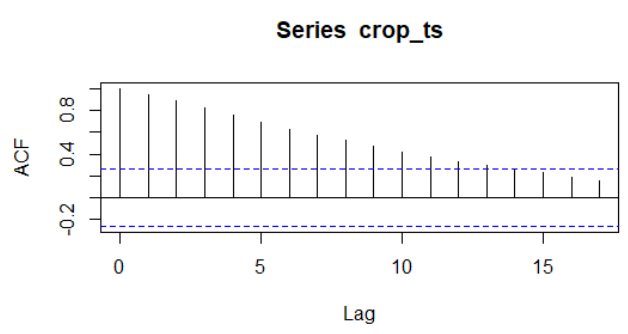

Figure 20: ACF of crop production

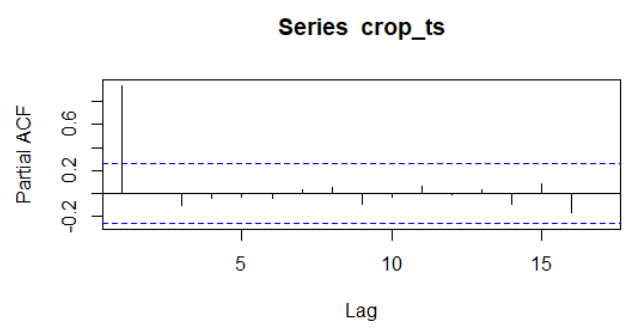

Figure 21: PACF of crop production

In above figure 20 and 21 we saw the $\mathrm{ACF}$ are above than blue lines and the PACF single line are above the blue line. So differentiate the data and check the adf test and also the ACF and PACF. Now calculate the autocorrelation function and partial autocorrelation function of residuals, the lines are below the blue dotted line (figure $22 \& 23$ ).
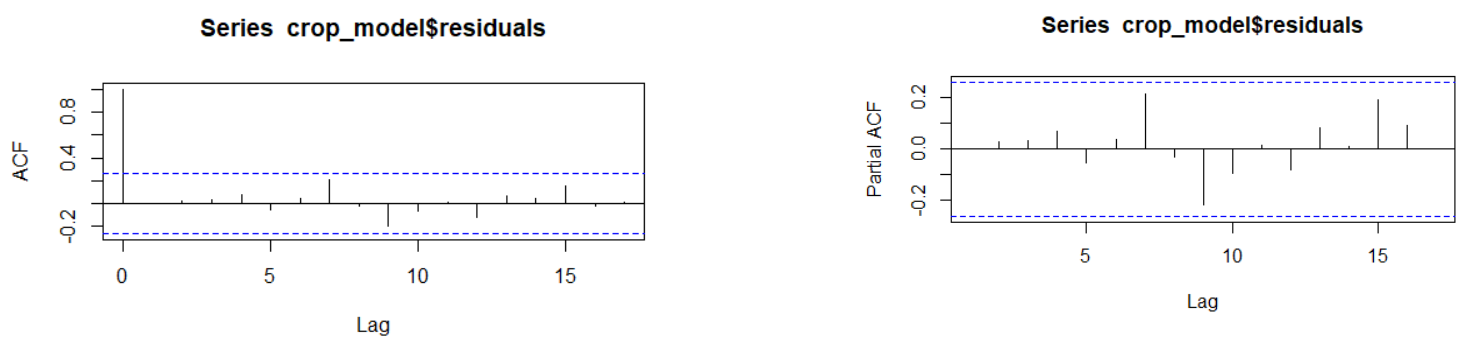

Figure 22: ACF of crop production residual Figure 23: PACF of crop production residual

Again we calculate the adf.test, the p-value is 0.02294 is lower than 0.05 so the data is stationary. Plot the graph to see the result (figure 24). If the data is stationary means the mean and variance are constant throughout the period. And use the forecast in build function to predicting the future.

R Code: 


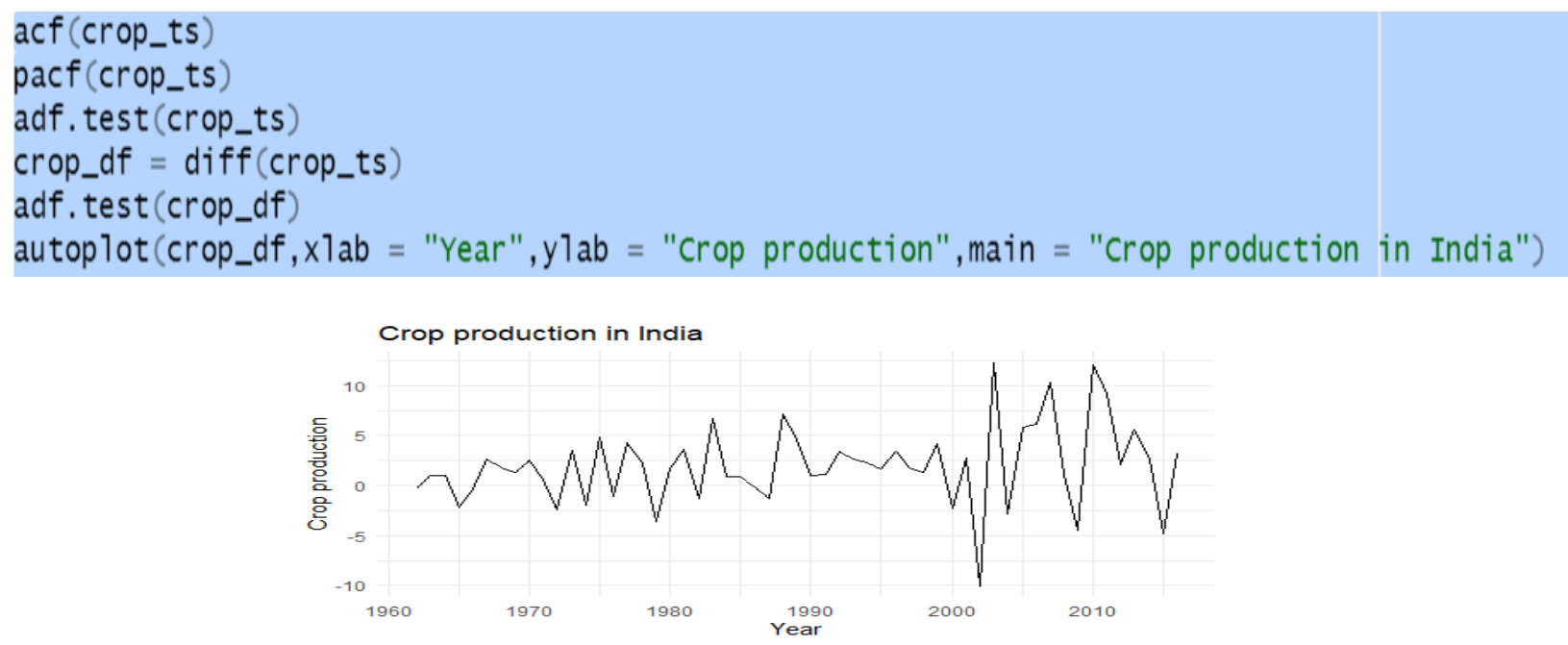

Figure 24: Graph of crop production (Stationary)

We can use the auto Arima function to calculate the $\mathrm{p}, \mathrm{d}$ and $\mathrm{q}$. Suppose we manually calculate the $p, d$ and $q$ means the aic and bic values are higher when compared to auto Arima function. The Akaike Information Criteria (AIC) and Bayesian Information Criteria (BIC) values are least value when compared to others. That means the $\mathrm{p}, \mathrm{d}$, q values are best fit to predicting the future. The $\mathrm{p}, \mathrm{d}$, q value is $(1,1,0)$ is the best fit for forecasting and the AIC value is 310.61 and the BIC value is 316.63 . We use the 90 per cent level of significant to forecasting for next 25 years from 2017 to 2041 (figure 25).

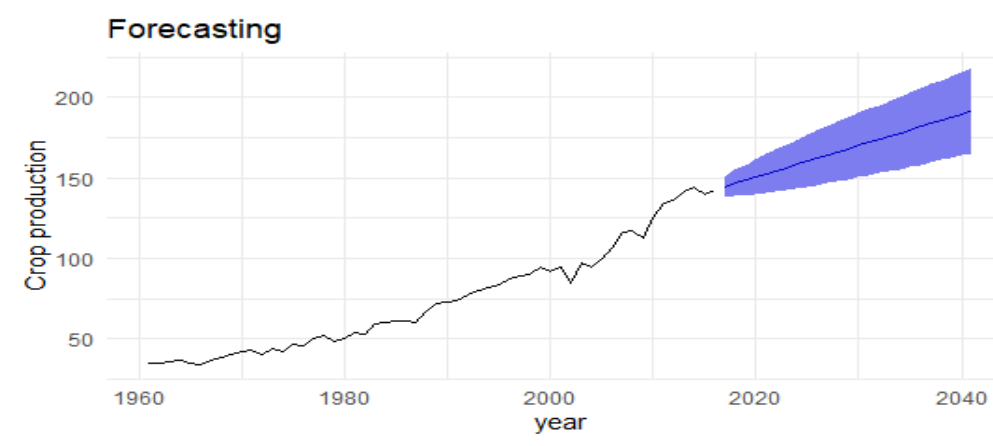

Figure 25: Crop Production Forecasting

We conclude that as the year increases, the crop production in India also gradually increases.

\section{Area of land Forecasting}

Import the area of land dataset in RStudio to predict the future area of land used by agriculture of India for next 25 years. And check the class of data, if the dataset are not in time series change the data into time series. Import some packages like forecast and time series; it's useful for forecasting in R.

\begin{tabular}{|l|l|}
\hline Minimum & 17.50 \\
\hline $1^{\text {st }}$ Quarter & 17.95 \\
\hline
\end{tabular}




\begin{tabular}{|l|l|}
\hline Median & 18.01 \\
\hline Mean & 17.98 \\
\hline $3^{\text {rd }}$ Quarter & 18.10 \\
\hline Maximum & 18.16 \\
\hline
\end{tabular}

Plot the data in auto plot function (figure 26), we can check the data is stationary or nonstationary. If the data is non stationary convert the data into stationary and then proceed to next step. The area of land used by agriculture data is non stationary we can find by using the Augmented Dickey-Fuller test shortly called as adf.test. The p-value is 0.5934 greater than 0.05 means the data is non-stationary.

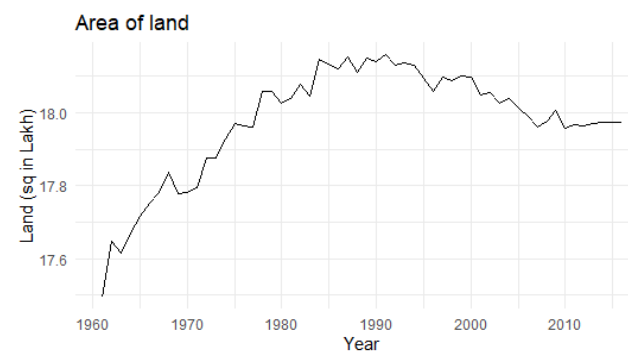

Figure 26: Graph of area of land used by agricultural

We can detect that the data is stationary or not using the ACF (Autocorrelation Function) and PACF (Partial Autocorrelation Function). If the graph is above and below the blue line, the data is non-stationary.

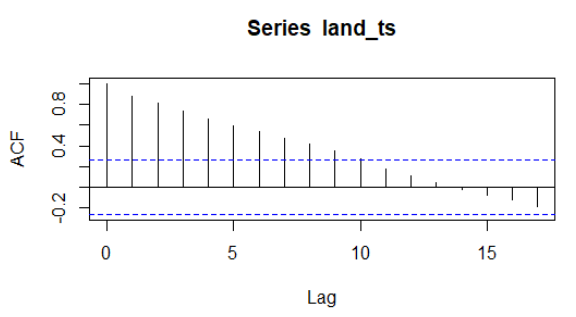

Figure 27: ACF of area of land

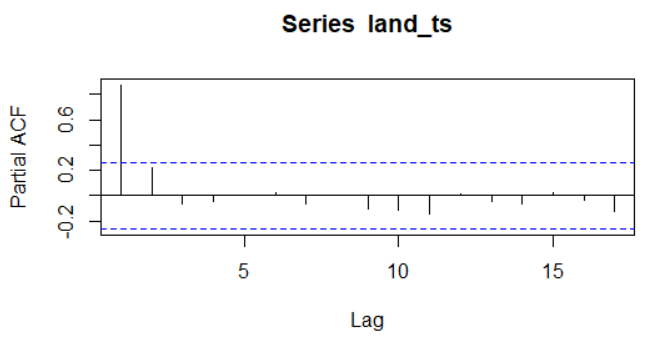

Figure 28: PACF of area of land

In above figure 27 and 28 we saw the ACF and PACF are above than blue lines. So differentiate the data and check the adf test and also the ACF and PACF. Now calculate the autocorrelation function and partial autocorrelation function of residuals, the lines are below the blue dotted line (figure $29 \& 30$ ). 


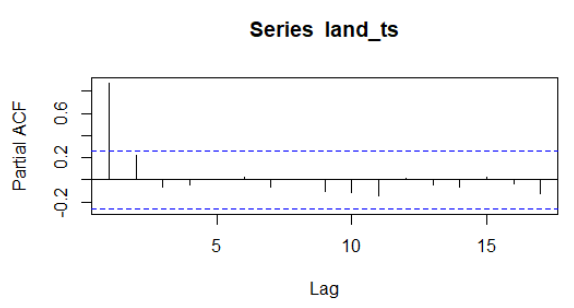

Figure 29: ACF of area of land residual

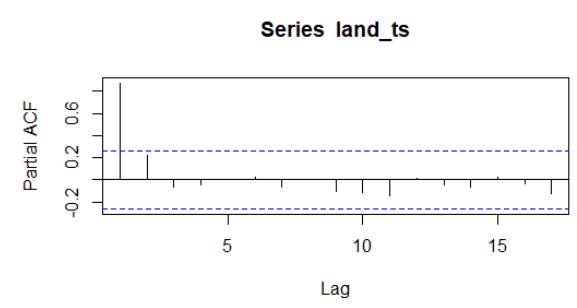

Figure 30: PACF of area of land residual

Again we calculate the augmented dickey-fuller test the p-value is 0.02235 is lower than 0.05 so the data is stationary. Plot the graph to see the result (figure 31). If the data is stationary means the mean and variance are constant throughout the period. And use the forecast in build function to predicting the future.

R Code:

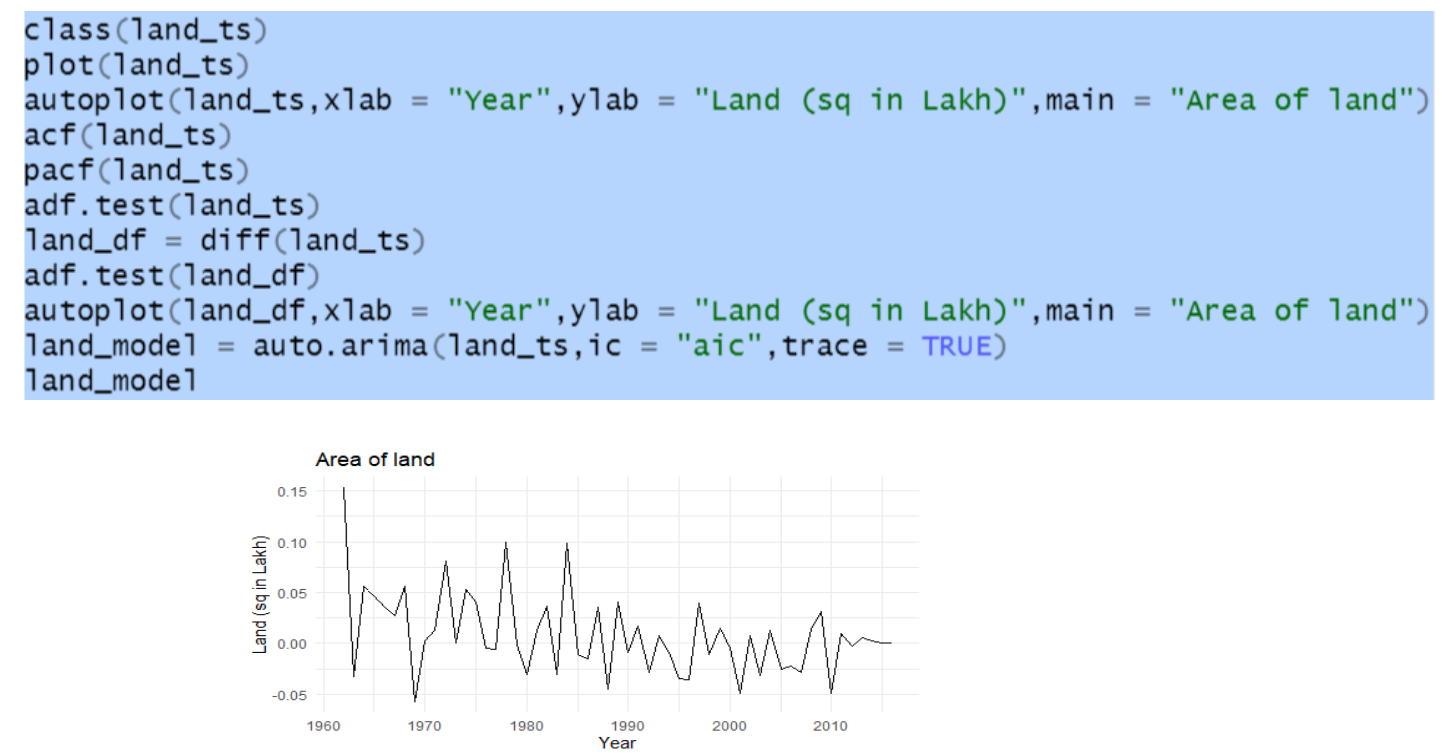

Figure 31: Graph of Area of land used by agricultural (Stationary)

We can use the auto arima function to calculate the $\mathrm{p}, \mathrm{d}$ and $\mathrm{q}$. Suppose we manually calculate the $\mathrm{p}, \mathrm{d}$ and $\mathrm{q}$ means the aic and bic values are higher when compared to auto arima function. The Akaike Information Criteria (AIC) and Bayesian Information Criteria (BIC) values are least value when compared to others. That means the $\mathrm{p}, \mathrm{d}$, $\mathrm{q}$ values are best fit to predicting the future. We use the 90 per cent level of significant to forecasting for next 25 years (figure 32). The (p,d,q) value is $(2,2,2)$ and the AIC value is -195.83 and the BIC value is -185.89 is best fit for prediction. 


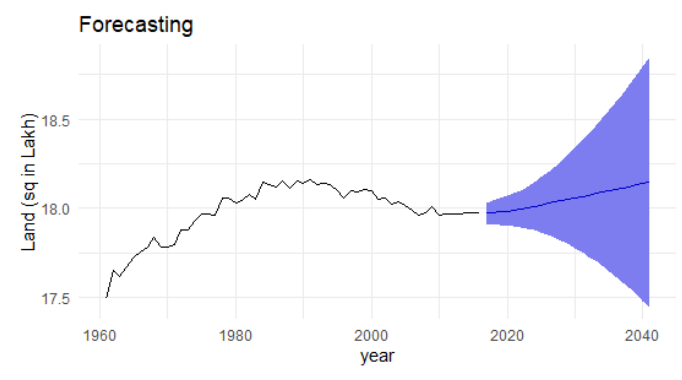

Figure 32: Area of land used by agricultural Forecasting

We conclude that the year is increases and the area of land used by agricultural in India is also slightly increases.

\section{Conclusion}

Since the temperature ranges from 24.66 to 25.57 degrees Celsius, the rainfall ranges from 93.95 to 97.18 millimeters, and the field area ranges from 17.97 to 18.15 square meters in lakh, we may infer that crop production over the next twenty-five years would steadily rise from 144.5 to 191.7 indexes. Despite our predictions, we can see that while individual population declines and therefore agricultural land decreases, crop demand for the mass population increases. Farmers use hybrid variety plants, seeds, and other goods to improve crop yield when operating with a restricted amount of ground. However, it uses a lot of water, and since rainfall is falling in contrast to previous years, underground water levels are falling as well, causing the crop to fade.

We need to raise awareness among farmers about the characteristics and volume of water that should be used for hybrid variety seeds in order to minimize the chance of destroying them all. We also need to raise awareness about crop insurance. Many real estate brokers and multinational corporations are attempting to enter the Agri land market in India. The government can amend the act to secure divide Agri land from private businesses. (For example, the government should encourage farmers to do only Agri in agro - based property, and the government should not help any organizations that generate revenue for the country.) The GDP rate would rise if the government invests in agriculture.

\section{Reference:}

[1] Chen, S., et al. "The time series forecasting: from the aspect of network." arXiv preprint arXiv: 1403.1713 (2014).

[2] Devi, B. Uma, D. Sundar, and P. Alli. "An Effective Time Series Analysis for Stock Trend Prediction Using ARIMA Model for Nifty Midcap-50."

[3] Box, George EP, and George C. Tiao. "Intervention analysis with applications to economic and environmental problems." Journal of the American Statistical Association 70.349 (1975):

70-79. 
[4] Kofi agyarko ababio, June 2012, "Comparative study of stock price forecasting using arima and arimax models".

[5] L---Stern Group Ly Pham, Time Series Analysis with ARIMA - ARCH/GARCH model in $R$

[6] Al Wadia, Mohd Tahir Ismail S, "Selecting Wavelet Transforms Model in Forecasting Financial Time Series Data Based on ARIMA Model", Applied Mathematical Sciences, Vol. 5, 2011 , no. 7, $315-326$

[7] Anderson, David Raymond. Model based inference in the life sciences: a primer on evidence. New York: Springer, 2008.

[8] Chatfield, Chris. The analysis of time series: an introduction. CRC press, 2013.

[9] Christodoulos, Charisios, Christos Michalakelis, and Dimitris Varoutas. "Forecasting with limited data: Combining ARIMA and diffusion models. "Technological forecasting and social change 77.4 (2010): 558-565.

[10] Pai, Ping-Feng, and Wei-Chiang Hong. "An improved neural network model in forecasting arrivals." Annals of Tourism Research 32.4 (2005): 1138-1141.

[11] Nayak, S. C., and B. B. Mishra. "A Neuro-Genetic Technique for Index Prediction.

[12] Liang, Jiuzhen, Wei Song, and Mei Wang. "Stock price prediction based on procedural neural networks." Advances in Artificial Neural Systems 2011 (2011): 6.

[13] Jarrett, Jeffrey E., and Eric Kyper. "ARIMA modeling with intervention to forecast and analyze chinese stock prices." International Journal of Engineering Business Management 3.3 (2011): 53-58.

[14] Yang, Yuhong. "Can the strengths of AIC and BIC be shared? A conflict between model indentification and regression estimation." Biometrika 92.4 (2005): 937-950.

[15] Tseng, Fang-Mei, et al. "Fuzzy ARIMA model for forecasting the foreign exchange market." Fuzzy sets and systems 118.1 (2001): 9-19.

[16] Contreras, J., Espinola, R., Nogales, F. J., \& Conejo, A. J. (2003). ARIMA models to predict next-day electricity prices. Power Systems, IEEE Transactions on, 18(3), 1014-1020.

[17] Willmott, Cort J., and Kenji Matsuura. "Advantages of the mean absolute error (MAE) over the root mean square error (RMSE) in assessing average model performance." Climate Research 30.1 (2005): 79.

[18] A. Pankratz, Forecasting with Dynamic Regression models, Wiley Interscience, 1991.

[19] http://www.nseindia.com/products/content/equities/equities/eq_security.htm. 
[20] $R$ Development Core Team (2008). $R$ : A language and environment for statistical computing. $R$ Foundation for Statistical Computing, Vienna, Austria. ISBN 3-900051-07-0, URL http://www.Rproject.org. 